\title{
Prevalence and usage pattern of dietary supplements by individuals with features of metabolic syndrome
}

\author{
R. Akilen ${ }^{1}$, A. Tsiami ${ }^{1}$, D. Devendra ${ }^{2}$ and N. Robinson ${ }^{1}$ \\ ${ }^{1}$ Faculty of Health and Human Sciences, Thames Valley University, UK and ${ }^{2}$ Department of Investigative Sciences, Faculty \\ of Medicine, Imperial College London and Brent NHS, UK
}

Background: Individuals with specific health conditions, like features of metabolic syndrome (FeMS) may use range of dietary and herbal supplements and other complementary and alternative approaches in order to improve their health status. The objective of this study is to determine whether individuals with self reported FeMS were more likely to use different dietary supplements compared with individuals without self reported FeMS. Furthermore, this study will provide preliminary and exploratory data for future studies.

Methods: Using a cross-sectional study design, information was obtained by online and self-administered questionnaires from a random sample of the members of Thames Valley University (TVU), London, UK. The study protocol was approved by the TVU research ethics committee. A total of 300 individuals were invited to participate in this study, of this 210 completed the questionnaires (giving a $70 \%$ response rate). The diagnosis of diabetes mellitus, hypertension, dyslipidaemia and obesity in this study was based on self reported clinically diagnosed medical conditions in hospitals. Therefore, diagnosis of FeMS was defined by any individuals having at least one self reported, clinically diagnosed medical condition of diabetes or hypertension or dyslipidaemia or obesity. Finally, two categories were created for cross-tabulation analysis; Individuals with and without FeMS. The questionnaire was developed by bearing in mind the requirements for cross tabulation and reporting. Cross tabulation yielded information about any correlations between dietary supplementation practices and FeMS. Chi-square test was employed to test whether any correlations reached statistical significance.

Results: Of the 210 individuals completed the study $32 \%$ ( $n$ 66) were currently using or had used dietary supplements in the past 12 months. The five most common dietary supplements used by the individuals currently or in the past 12 months were found to be; multi vitamins $(38 \% ; n 25)$, fish oils $(35 \% ; n 23)$, Ca $(26 \% ; n 17)$, different herbal supplements $(24 \% ; n 16)$ and omega-3 oils (24\%; $n 16)$. Individuals with FeMS were significantly more likely to use different dietary supplements compared with individuals without FeMS $(P<0.001)$. Among individuals with FeMS, older age ( $>55$ years) and higher educational attainment (undergraduate or post graduate) were independently associated with dietary supplement use. Furthermore, individuals with FeMS were significantly less likely to report or discuss the use of dietary supplements with their general practitioner $(P=0.043)$.

Discussion: There is a substantial increase in the use of dietary supplements and complementary and alternative medical therapies within the UK. As metabolic syndrome or diabetes mellitus is a chronic debilitating medical condition, there are two important areas for future research. Firstly, there is an urgent need to use rigorous research designs to establish the efficacy of several dietary supplements or herbal remedies that are currently being used by individuals with diabetes or metabolic syndrome. Secondly, future studies must determine the effectiveness of herbal remedies or diet supplements used in typical clinical situations and the effect of these herbal supplements should be assessed by quality randomised controlled clinical trials.

Conclusion: FeMS is an independent predictor of dietary supplement use. Dietary supplement use is more common in older individuals and those with more than high school education. This study also suggested that introducing dietary or herbal supplements or investigating the efficacy of any dietary supplements for individuals with FeMS is acceptable and feasible. 